\title{
Pulvermetallurgische Herstellung von innovativen Hochtemperaturwerkstoffen
}

\author{
Helmut Clemens ${ }^{1}$, Svea Mayer ${ }^{1}$ und Martin Heilmaier ${ }^{2}$ \\ 'Department Metallkunde und Werkstoffprüfung, Montanuniversität Leoben, Leoben, Österreich \\ ${ }^{2}$ Institut für Angewandte Materialien - Werkstoffkunde, Karlsruher Institut für Technologie (KIT), Karlsruhe, \\ Deutschland
}

Eingegangen 11. Januar 2018; angenommen 13. Januar 2018; online publiziert 5. Februar 2018

\begin{abstract}
Zusammenfassung: Die pulvermetallurgische Herstellung von Bauteilen aus innovativen Werkstoffen, wie intermetallischen Titanaluminiden und Refraktärmetallsiliziden, bietet eine Vielfalt von Anwendungsmöglichkeiten in modernen Flugzeugtriebwerken und Verbrennungsmotoren. Für die Produktion von hochwertigen Legierungspulvern und deren Verarbeitung stehen mehrere industriell relevante Verfahren bereit.
\end{abstract}

Schlüsselwörter: Hochtemperaturwerkstoffe, Pulvermetallurgie, Pulverherstellung, Verarbeitung

Powder Metallurgical Production of Innovative HighTemperature Materials

Abstract: Powder metallurgical production of structural parts made of innovative materials such as intermetallic titanium aluminides and refractory metal silicides offer a variety of applications in advanced aero-engines and automotive combustion engines. For the production of high grade alloy powders and their further processing several methods have been developed to industrial scale.

Keywords: High-temperature materials, Powder metallurgy, Powder production, Processing

\section{Einleitung}

Moderne Flugtriebwerke für zivile Anwendungen müssen höchsten Ansprüchen im Hinblick auf Zuverlässigkeit, Gewicht, Leistung, Wirtschaftlichkeit und Lebensdauer gerecht werden. Im Laufe der letzten Jahrzehnte wurden

\footnotetext{
Univ.-Prof. Dipl.-Ing. Dr. mont. H. Clemens ( $\varangle)$ Department Metallkunde und Werkstoffprüfung, Montanuniversität Leoben,

Roseggerstraße 12,

8700 Leoben, Österreich

helmut.clemens@unileoben.ac.at
}

Triebwerke entwickelt, die die Marktanforderungen erfüllen und ein hohes Maß an technischer Perfektion erreicht haben. Gleichzeitig konnten Lärmpegel und Schadstoffemissionen erheblich reduziert werden. Bezüglich aktueller Entwicklungstrends im Triebwerksbau wird der Leser auf die jeweilige Homepage der führenden Triebwerkshersteller, wie General Electric, Pratt \& Whitney, Rolls Royce, Safran, etc., verwiesen. Was heute im Triebwerksbau Stand der Technik ist, ist neben den Verbesserungen der Auslegung sowie der Aero- und Thermodynamik in nicht unerheblichem Maß den Fortschritten auf den Gebieten der Werkstoff-, Fertigungs- und Oberflächentechnik zu verdanken. Wegen des ausgereiften Designs der Triebwerke findet der Wettbewerb zukünftig über innovative Werkstoffe und Herstellverfahren statt [1]. Dies stellt sowohl die Werkstofftechnik als auch die Fertigungstechnik vor neue Herausforderungen. Die klassischen Werkstoffe für Triebwerksbauteile, wie Titan- und Nickellegierungen, haben im Laufe der letzten Jahrzehnte ein hohes Maß an technischer Perfektion erreicht. Ihr Verbesserungspotential ist jedoch aus verschiedenen hier jedoch nicht diskutierten Gründen begrenzt.

Neue Werkstoffe und Bauweisen sind derzeit in der Entwicklung, was eine Leistungsverbesserung des Triebwerkes ermöglicht. In Abb. 1 ist die spezifische Festigkeit (Zugfestigkeit/Dichte) von herkömmlichen und neuen Werkstoffen über der Temperatur aufgetragen. Zwei vielversprechende Gruppen unter den neuen Werkstoffen für künftige Triebwerkgenerationen sind intermetallische Werkstoffe und Verbundwerkstoffe auf Basis von Refraktärmetallsiliziden. Intermetallische Titanaluminide auf Basis der geordneten $\gamma$-TiAl Phase haben sich in den letzten Jahren als innovative Leichtbauwerkstoffe für Betriebstemperaturen bis ca. $750^{\circ} \mathrm{C}$ für Luftfahrt- und Automobilanwendungen etabliert. Besonders die geringe Dichte von wenig mehr als $4 \mathrm{~g} / \mathrm{cm}^{3}$ macht diesen Werkstoff für rotierende Teile, wie Turbinenschaufeln und Turboladerlaufräder, interessant [2].

Verbundwerkstoffe haben entweder eine Polymer-, Metall-, Intermetall- oder eine Keramikmatrix, verstärkt 
Abb. 1: Spezifische Zugfestigkeit in Abhängigkeit von der Anwendungstemperatur für potentielle Triebwerkswerkstoffe

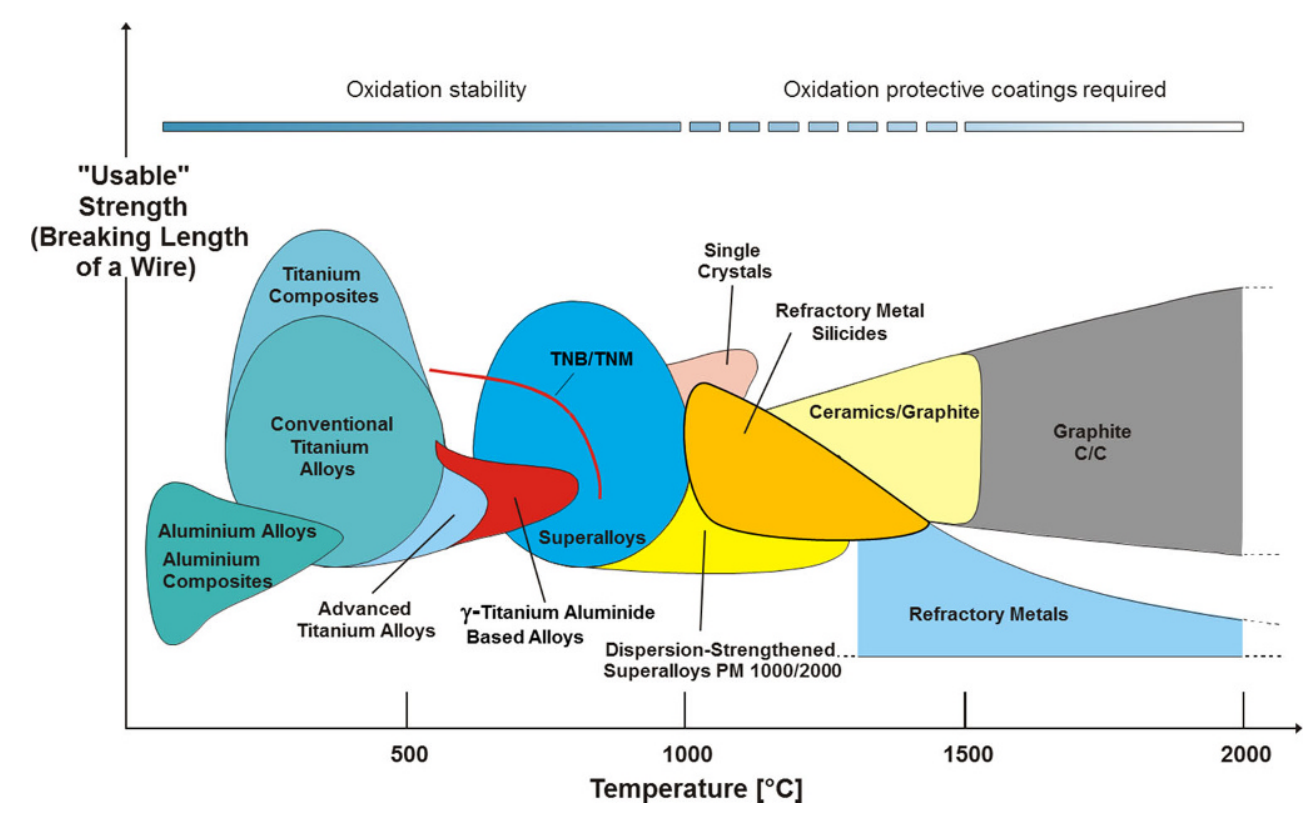

durch mechanisch und thermisch stabile Teilchen, Kurzfasern oder Langfasern. Mit hochfesten Verbundwerkstoffen auf Basis der Refraktärmetallsilizide kann das Gewicht von Triebwerksbauteilen, bei gleichzeitig höherem Temperaturpotential von bis zu $200^{\circ} \mathrm{C}$, um bis zu $50 \%$ gegenüber herkömmlichen Nickel Superlegierungen reduziert werden [3].

Während in den letzten Jahrzehnten vorrangig schmelzmetallurgische Prozesse zur Herstellung von hochwertigem Vormaterial entwickelt wurden, welches in weiterer Folge durch Feinguss bzw. Warmumformung zu Bauteilen verarbeitet wurde, sind pulvermetallurgische Herstellverfahren auf Nischenanwendungen, wie z. B. spezielle NickelbasisSuperlegierungen für Turbinenscheiben, begrenzt.

Durch die Einführung von pulverbasierten Fertigungsmethoden, wie z. B. das bereits verwendete endkonturnahe heiß-isostatische Pressen von pulvergefüllten Kapseln (engl. Hot Isostatic Pressing, HIP), der Metallpulverspritzguss (engl. Metal Injection Molding, MIM) und die Generative Fertigung (engl. Additive Manufacturing, AM), wurde das Interesse an der Herstellung von hochwertigem Pulver aus Hochleistungswerkstoffen neu belebt.

\section{Intermetallische Titanaluminide}

\subsection{Zusammensetzung von TiAl Legierungen}

Die Zusammensetzung von $\gamma$-TiAl-Basislegierungen kann wie folgt zusammengefasst werden, wobei die Legierungsgehalte in diesem Artikel in Atomprozent (at\%) angegeben sind: $\mathrm{Ti}-(42-48) \mathrm{Al}-(1-3) \times 1-(1-10) \times 2-(0-1) \times 3$, wobei $\mathrm{X} 1=\mathrm{Cr}$, $\mathrm{Mn}, \mathrm{V}, \mathrm{X} 2=\mathrm{Nb}, \mathrm{Ta}, \mathrm{W}$, Mo und X3=Si, B, C, Seltenerdmetalle $[2,4-6]$.

Je nach Menge der Legierungselemente und dem Entwicklungsjahr kann man $\gamma$-TiAl-Basislegierungen bestimmten Legierungsgenerationen zuordnen. Die ersten TiAlLegierungen enthielten nur geringe Mengen ( $<3$ at\%) ei- nes Legierungselements, z. B. V, Nb oder $\mathrm{Cr}$, und werden daher als Legierungen der 1. Generation bezeichnet. Ein Beispiel stellt die Legierung Ti-48Al-2 $\mathrm{Cr}$ dar. Legierungen der 2. Generation wurden schon mehrere Elemente hinzulegiert, um den gekoppelten Effekt dieser auszunutzen. Beispiele sind hierfür die von General Electric (USA) entwickelte Ti-48Al-2Nb-2Cr-Legierung, die als Gusslegierung für Turbinenschaufeln in Flugzeugtriebwerken eingesetzt wird [6], oder die von Plansee (Österreich) auf den Markt gebrachte Ti-46.5Al-4(Cr,Nb,Ta,B)-Legierung [7]. Legierungen der 3. Generation weisen meist einen hohen Gehalt an Legierungselementen auf, damit spezielle Eigenschaften deutlich verbessert werden. Beispielhaft sind hier die vom Helmholtz-Zentrum Geesthacht (HZG), Deutschland, entwickelten TNB-Legierungen, die auf der folgenden $\mathrm{Zu}$ sammensetzung beruhen: Ti-45Al-(5-10) Nb-(0-1)(C,B). Dieser Legierungstyp zeichnet sich durch eine hohe Zug- und Kriechfestigkeit sowie eine verbesserte Oxidationsbeständigkeit aus [2]. Durch Zugabe von geringen Mengen an C kann in diesen Legierungen eine wirkungsvolle Ausscheidungshärtung durch Karbide erzielt werden, was sich besonders auf die Kriechfestigkeit auswirkt. Eine neuere Legierungsklasse, die man ebenfalls der 3. Generation zuordnet, stellt die so genannte TNM-Legierung dar, die im Rahmen eines langfristigen Projekts für die Anwendung als Schmiedelegierung für Turbinenschaufeln im Flugzeugtriebwerk entwickelt wurde [4]. Die Abkürzung TNM leitet sich aus der Legierungszusammensetzung ab:

Ti-(42-45)Al-(3-5) Nb-(0.1-2)Mo-(0.1-0.2)B.

\subsection{Herstellung hochwertiger TiAl-Legierungs- pulver}

Die Herstellung von TiAl-Pulver über die Verdüsung der Schmelze, so genannte „Atomization Techniques“, ist technologisch herausfordernd: einerseits wegen des hohen Schmelzpunktes (ca. $1450^{\circ} \mathrm{C}$ ) und andererseits soll der 


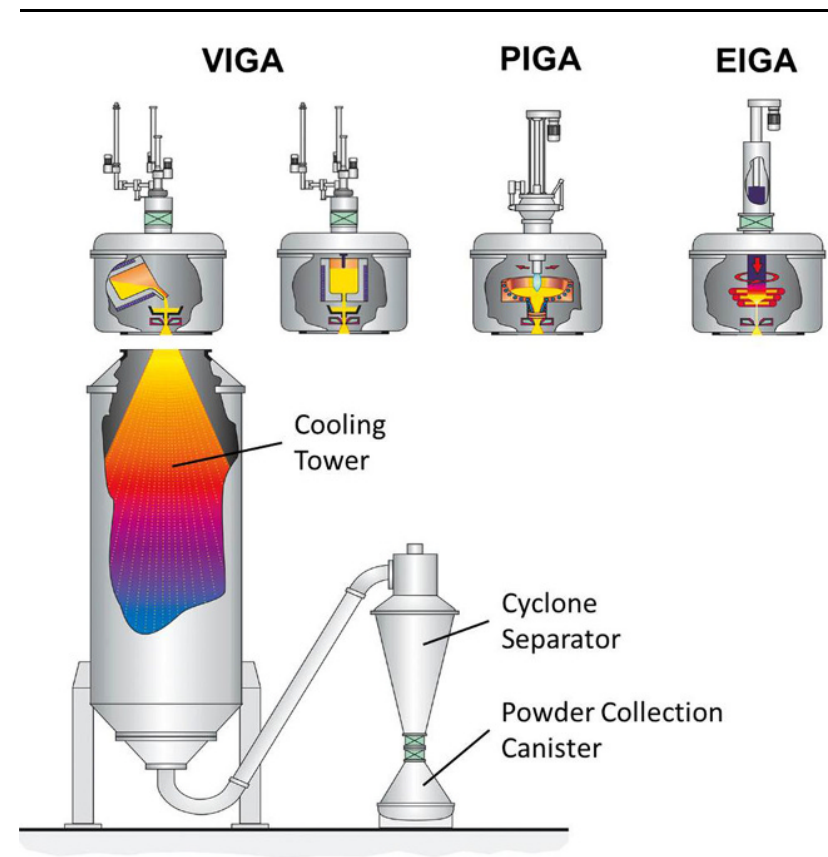

Abb. 2: Herstellungsprozesse für hochwertige TiAl-Pulver (siehe Text). Die Abbildung wurde von ALD Vacuum Technologies $\mathrm{GmbH}$, Hanau, Deutschland, zur Verfügung gestellt [9]

Prozess "keramikfrei“ unter Inertgas bzw. Vakuum durchgeführt werden [8]. Das liegt daran, dass die Schmelze sehr reaktiv ist und nahezu mit jedem Tiegelmaterial reagiert. Die Verdüsung der Schmelze erfolgt unter Inertgas, vorrangig Argon, da die Pulverpartikel, wegen ihrer hohen Affinität zu interstitiellen Verunreinigungen, empfindlich auf die Aufnahme von Sauerstoff, Stickstoff und Kohlenstoff sind. Aus diesem Grund dürfen TiAl-Pulver selbst bei Raumtemperatur nicht Luft ausgesetzt werden, da dies zu einem unerwünschten Anstieg der Sauerstoffkonzentration führt. An dieser Stelle sei angemerkt, dass in $\gamma$-TiAlBasislegierungen der Gehalt an Sauerstoff und Stickstoff $1000 \mu \mathrm{g} / \mathrm{g}$ nicht überschreiten soll, da es sonst zu einer Materialversprödung kommt, die sich im Zugversuch durch Versagen im elastischen Bereich äußert.

Als Herstellmethoden für hochwertige TiAl-Pulver wurden unterschiedliche Verfahren entwickelt, wie sie in Referenz [8] ausführlich beschrieben sind. Im Folgenden wird aus Platzgründen nur auf drei Verfahren eingegangen, die schematisch in Abb. 2 dargestellt sind [9]. Diese Verfahren sind zur Pulverherstellung im industriellen Maßstab geeignet. Hierbei handelt es sich um das VIGA-, PIGA- und EIGAVerfahren. VIGA steht für Vacuum Induction Melting combined with Inert Gas Atomization, PIGA für Plasma Melting Induction Guiding Gas Atomization und EIGA für Electrode Induction Melting Gas Atomization.

Der VIGA- und PIGA-Prozess gehört zu den Kaltwandtiegel-Verfahren. Beim VIGA-Prozess wird in einem wassergekühlten Kupfertiegel die Schmelze mittels Induktionsheizung flüssig gehalten. Wegen der Wasserkühlung bildet sich auf der Tiegeloberfläche ein sogenannter "Skull" aus TiAl, der die Schmelze vor Verunreinigung durch geIöstes Kupfer schützt. Auch beim in Referenz [8] beschriebenen PIGA-Verfahren wird ein wassergekühlter Kupfertie- gel verwendet, nur wird zur Beheizung ein Plasmabrenner eingesetzt. Während beim VIGA-Verfahren der Tiegel gekippt wird (bzw. die Düse durch eine Induktionsspule "geöffnet" "wird), die Schmelze dadurch in eine Düse fließt und danach den Verdüsungsraum betritt, wird beim PIGA-Verfahren ebenfalls mittels einer Induktionsspule die Düse geöffnet und der Schmelzstrahl tritt direkt in die Verdüsungskammer. Bei beiden Verfahren wird dann der Schmelzstrahl unter Druck mit gasförmigem Argon zerstäubt. Beide Verfahren zeichnen sich durch chemisch sehr homogene Pulver aus. Beim EIGA-Verfahren hingegen wird ein stabförmiger TiAl-Ingot in einer Induktionsspule kontinuierlich aufgeschmolzen. Der so erzeugte Schmelzstrom wird wiederum mit Inertgas verdüst. Ein Nachteil dieses Verfahrens liegt jedoch darin, dass sich im Stab befindliche Seigerungen auf das Pulver übertragen können [8]. Pulver, das durch Gasverdüsung eines Schmelzstrahls erzeugt wird, zeigt eine sphärische Teilchenform. Wegen der sehr hohen anfänglichen Kühlrate bei der Erstarrung der Teilchen, nach Referenz [8] ca. $2 \cdot 10^{5} \mathrm{~K} / \mathrm{s}$ für ein $45 \mu \mathrm{m}$ großes Pulverteilchen, liegt nach der Erstarrung ein sehr feinkörniges und homogenes Gefüge vor, dessen Phasenanteile weit vom thermodynamischen Gleichgewicht entfernt sein können $[8,10]$. Da die auftretenden Seigerungen nur an den Korngrenzen sind, können sie durch den anschließenden Kompaktierungsprozess, z.B. durch HIPen, effektiv beseitigt werden. Bei der Wahl der HIP-Parameter ist darauf zu achten, dass die ehemaligen Pulverkorngrenzen nicht mehr erkennbar sind, da sie mit Sauerstoff angereicherte Zonen darstellen. Das Gefüge ist dann feinkörnig und sehr homogen, und die auftretende Phasenfraktion entspricht jener des thermodynamischen Gleichgewichts [10]. An dieser Stelle sei angemerkt, dass die Gefügehomogenität geHIPter TiAl-Pulver deutlich höher ist als jene von gegossenen und geschmiedeten TiAlLegierungen. Dieser Vorteil sollte sich in einer vergleichbar höheren Zugbruchdehnung bei Raumtemperatur bemerkbar machen, was aber durch die prozessbedingte höhere Verunreinigung mit Sauerstoff verhindert wird.

Der Sauerstoff- und Stickstoffgehalt als Funktion der Pulvergröße ist für eine $\mathrm{Ti}-45 \mathrm{Al}-7.5 \mathrm{Nb}$-Legierung untersucht worden [8]. Das Ergebnis dabei ist, dass der Sauerstoffgehalt mit zunehmender Pulvergröße in den Bereich des Gussmaterials kommt, was für die mechanischen Eigenschaften von Vorteil wäre. Es hat sich aber gezeigt, dass bei größeren Pulverteilchen Poren auftreten, die mit Argon gefüllt sind [7, 8]. Das in TiAl nicht lösliche Argon wird beim HIP-Prozess zusammengepresst, so dass keine Porosität erkennbar ist. Bei anschließenden Wärmebehandlungen bzw. mechanischer Belastung bei hoher Temperatur können diese Poren aber wieder aufgehen, was mit thermisch induzierter Porosität (engl. Thermally Induced Porosity, TIP) bezeichnet wird. Es konnte gezeigt werden, dass bei TiAl-Blechen, die aus pulvermetallurgischem Vormaterial hergestellt wurden, die superplastischen Eigenschaften durch TIP drastisch reduziert wurden [7]. 
Abb. 3: Verarbeitungsrouten für TiAl-Legierungspulver; HIP: heiß-isostatisches Pressen; SPF: Superplastic Forming; DB: Diffusion Bonding [11]
Powder Metallurgy (PM)

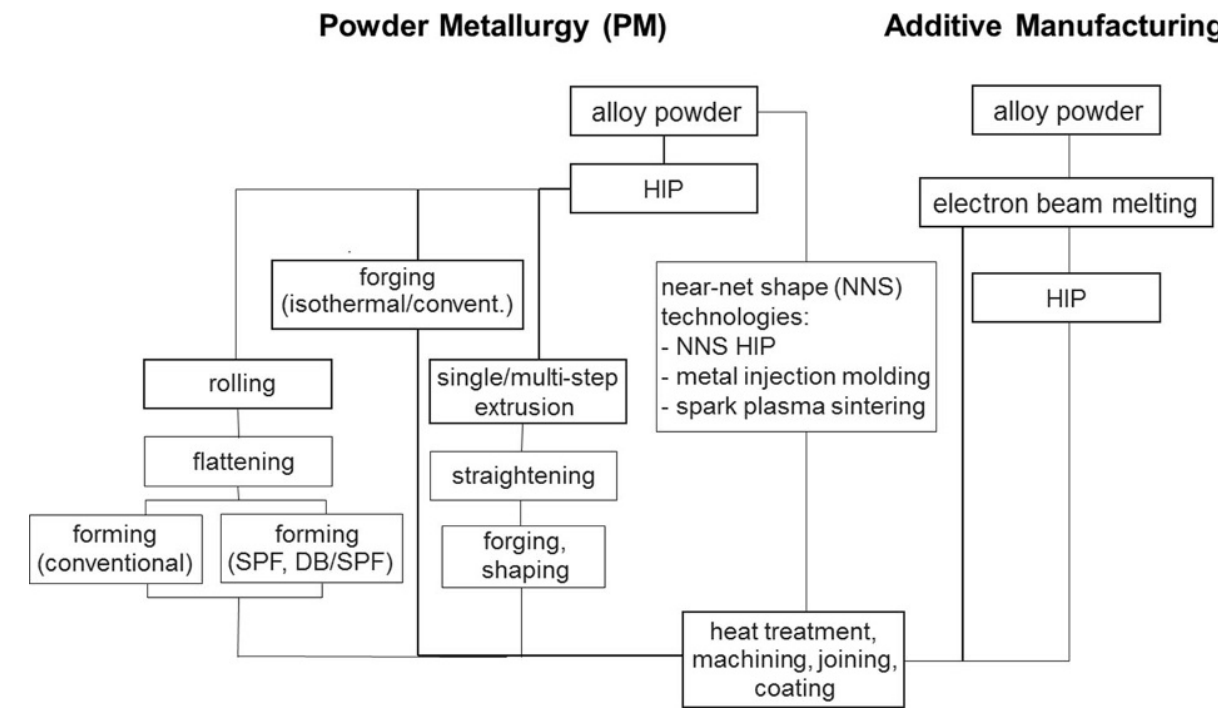

Additive Manufacturing (AM)

\subsection{Herstellung von Halbzeugen und Bauteilen}

Die potentiellen Verarbeitungsrouten für TiAl-Legierungspulver werden in Abb. 3 dargestellt [11]. GeHIPtes TiAl-Legierungspulver wurde als Vormaterial für das Walzen von Blechen eingesetzt. Dabei wurde ein sogenannter quasiisothermer Walzprozess entwickelt, der auf die schwierige Verarbeitbarkeit dieser intermetallischen Werkstoffklasse ausgelegt war [7]. Durch Einsatz von geHIPtem Vormaterial konnten TiAl-Bleche mit einer Abmessung von $2000 \times 500 \times 1 \mathrm{~mm}^{3}$ hergestellt werden. Bauteile, die aus diesen Blechen gefertigt wurden, wurden erfolgreich im Rahmen des US-Programms "High Speed Civil Transport" getestet [12]. Des Weiteren wurde zylinderförmig geHIPtes Vormaterial zum Schmieden von TiAl-Scheiben verwendet [4, 8]. Als Beispiele für Near-Net-Shape (NNS)-Techniken werden im Folgenden drei Verfahren erwähnt. Das HIPen von TiAl-Pulver in speziell gefertigten Kapseln diente der Herstellung endformnaher TiAl-Teile, die in weiterer Folge zu Ventilen für die Automobilanwendung verarbeitet wurden [7]. Des Weiteren wird diese Technik gegenwärtig evaluiert, ob sie sich zur Herstellung von Komponenten für Flugzeugtriebwerke eignet [13]. Eine Gruppe in Frankreich hat gezeigt, dass durch Spark Plasma Sintern (SPS) die endformnahe Herstellung von TiAl-Kompressorschaufeln möglich ist [14]. Bei diesem Verfahren erhält man vollkommen dichtes Material, wobei die Gefügeeinstellung und dadurch die mechanischen Eigenschaften von der Wahl der Sintertemperatur und der Abkühlrate abhängen. Ein „Upscaling" des Prozesses zur Herstellung von großen Niederdrucklaufschaufeln würde aber eine große technologische Herausforderung darstellen. Ein weiteres Verfahren, welches für die Herstellung von TiAl-Bauteilen in Erprobung ist, ist der MIM-Prozess, wie in [8] beschrieben. Wegen des druckfreien Sinterns und der hohen Prozesstemperatur weist das Bauteil ein grobes Gefüge sowie eine Restporosität auf. Des Weiteren ist die Aufnahme von interstitiell gelösten Verunreinigungen beim so genannten "Entbindern" ein Problem, da es zu einer Materialversprödung führt. Der MIM-Prozess hat den Vorteil, dass man relativ kostengünstig komplex geformte Bauteile herstellen kann. Er wird in Abschn. 3.3 am Beispiel der Refraktärmetallsilizide noch näher vorgestellt.

In den letzten Jahren haben sich sogenannte AM-Verfahren am Markt etabliert. Ein Verfahren, welches für die Verarbeitung von TiAl-Pulver besonders attraktiv ist, ist das Electron Beam Melting (EBM) [15, 16]. Das EBM-Verfahren zählt zu den pulverbettbasierten Strahlschmelzverfahren. Als Energiequelle wird der Elektronenstrahl nicht nur zum selektiven Schmelzen einer oder mehrerer Pulverschichten des Werkstoffs genutzt, sondern auch zum Vorheizen des Pulverbetts. Aufgrund der hohen Energiedichte kann TiAl prinzipiell voll verdichtet werden. Wegen des Elektronenstrahls ist die Prozessatmosphäre Vakuum, was dem äußerst reaktiven TiAl zugutekommt. Die Strahlablenkung erfolgt trägheitsfrei mittels eines elektromagnetischen Linsensystems, wodurch vergleichsweise hohe Scangeschwindigkeiten und Bauraten erreicht werden können [16].

Im Folgenden wird auf die wesentlichen EBM-Verfahrensschritte eingegangen: 1) Erstellung eines dreidimensionalen CAD (engl. Computer-Aided Design) Modells des zu fertigenden Bauteils; 2) Zerlegung dieses Modells in zweidimensionale Schnitte; 3) Aufbringen einer dünnen ebenen TiAl-Pulverlage aus Vorratsbehältern auf eine Plattform; 4) Thermische Behandlung der Pulverschicht, d.h. Vorheizen des Pulverbetts und nachfolgendes selektives Aufschmelzen basierend auf den Daten des jeweiligen 2DSchnitts; 5) Absenken der Plattform um eine Pulverlage; 6) Wiederholung der Schritte 3 ) bis 5 ) bis zum letzten zweidimensionalen Schnitt. Nach Abschluss des Bauvorgangs erfolgen die Entnahme des Bauteils sowie die Entfernung überschüssigen Pulvers und eventuell notwendiger Stützstrukturen. Mittels dieser Methode konnten aus TiAl-Pulver bereits Prototypen von Turboladerlaufrädern und Turbinenschaufeln hergestellt werden [15-17]. Obwohl diese Technik noch relativ neu ist, sollen bereits 2018 die ersten mittels EBM gefertigten TiAl-Schaufeln im Flugbetrieb getestet werden [18]. 
Fast allen in Abb. 3 beschriebenen Verarbeitungsrouten ist gemeinsam, dass das Gefüge nach der Verarbeitung relativ fein vorliegt, was im Fall einer Hochtemperaturanwendung zu inakzeptablen Kriecheigenschaften führt. Aus diesem Grund müssen die Bauteile einer geeigneten Wärmebehandlung unterzogen werden. Generelles Ziel ist es, ausgewogene mechanische Eigenschaften zu erzielen, d.h. gute Hochtemperaturfestigkeit bei gleichzeitig genügender Bruchdehnung und Bruchzähigkeit bei Raumtemperatur. Der Zusammenhang von Gefüge und mechanischen Eigenschaften in TiAl-Legierungen ist ausführlich in [2, 4] beschrieben.

\section{Refraktärmetallsilizide}

\subsection{Zusammensetzung}

Aus ökonomischen sowie Leistungsgründen ist man bestrebt, die Verbrennungstemperaturen in Turbinen weiter zu erhöhen. Will man den Einsatzbereich von Hochtemperaturwerkstoffen über die derzeitige von den Nickel-Superlegierungen gegebene Grenze von etwa $1100^{\circ} \mathrm{C}$ ausdehnen, so verbleiben nach Abb. 1 neben den Keramik-basierten Materialien als Metall-basierte Werkstoffe die so genannten Refraktärmetall-(RM-)Silizide, die sich gegenüber den klassischen Refraktärmetallen Mo, W, Nb und Ta aufgrund der Zugabe geeigneter intermetallischer Phasen zu einer Mischkristallmatrix durch einen adäquaten Oxidationswiderstand auszeichnen [19, 20]. Aus Gewichtsgründen kommen von den genannten Refraktärmetallen für den Einsatz in Flugturbinen lediglich Niob- oder Molybdän-Silizidlegierungen in Frage; allerdings bieten diese mit Schmelzpunkten von typisch jenseits 1700 bzw. $2000^{\circ} \mathrm{C}$ das Potential, die Arbeitstemperaturen in Gasturbinen um mindestens $200^{\circ} \mathrm{C}$ zu steigern. Im Gegensatz zu den intermetallischen $\gamma$-TiAIBasislegierungen befinden sich die hier vorgestellten Werkstoffe noch in der Anmeldung von Patenten beginnend in den späten 90er-Jahren durch Mitarbeiter von General Electric [20] bzw. Pratt \& Whitney [19] sowie auch im neuen Jahrtausend bei Plansee [21], d. h. am Anfang ihrer Entwicklung und haben noch keine Anwendungsreife erlangt. Hinsichtlich der bis dato untersuchten Zusammensetzungen ist beiden Legierungssystemen gemein, dass zur Erlangung akzeptabler Hochtemperaturoxidationswiderstände $\mathrm{Si}$ als Hauptlegierungselement in einem Konzentrationsbereich von 9 bis 20 at\% zugegeben wird. Dadurch entstehen neben dem jeweiligen Mo- bzw. Nb-Mischkristall, in dem Silizium nur in relativ geringem Maße löslich ist und der für eine akzeptable Duktilität bzw. Bruchzähigkeit bei Raumtemperatur sorgen soll, intermetallische Phasen vom Typ $\mathrm{RM}_{3} \mathrm{Si}$ oder $\mathrm{RM}_{5} \mathrm{Si}_{3}$. Da diese aufgrund der gewählten $\mathrm{Zu}-$ sammensetzungen durchaus in Volumenanteilen bis über $50 \%$ auftreten können, erklärt dies auch den heute in der Literatur häufig verwendeten Begriff „Refraktärmetallsilizidlegierungen“. Den RM-Siliziden fällt im Hinblick auf die Eigenschaften der Legierungen bei den avisierten hohen Einsatztemperaturen eine zweifache Aufgabe zu: (i) zum einen sollen sie als festigkeitssteigernde Fremdphasen das Kriechverhalten verbessern; dies gelingt jedoch nur dann, wenn sie hinreichend klein und dabei homogen im Gefüge verteilt sind $[20,22,23]$, (ii) zum anderen stellen sie das Si-Reservoir, das die Ausbildung einer in Luft schützenden $\mathrm{SiO}_{2}$-Schicht bewirken soll.

Bereits in Berzik's Patent [19] wurde nachgewiesen, dass die Kinetik der glasartigen $\mathrm{SiO}_{2}$-Schichtbildung für einen hinreichenden Schutz bei Temperaturen $>600^{\circ} \mathrm{C}$ zu langsam abläuft, weshalb aktuellen Mo-Si-Legierungen immer Bor (typisch um 8 at\%) zur Herabsetzung der Viskosität der aufwachsenden Glasschicht und damit zur Verbesserung ihrer Fließfähigkeit zugegeben wird. Im ternären System Mo(9-13)Si-(8-10)B (alle Zahlenabgaben in at.\%) stehen dann die drei Phasen Mo-Mischkristall, $\mathrm{Mo}_{3} \mathrm{Si}$ sowie $\mathrm{Mo}_{5} \mathrm{SiB}_{2}$ miteinander im Gleichgewicht. Mit der Zugabe von Bor wird dem Masseverlust durch Sublimation des Oxids $\mathrm{MoO}_{3}$ wirksam begegnet, kann anfänglich jedoch nicht vollständig vermieden werden. Auch Legierungen mit der oben genannten Phasenzusammensetzung zeigen daher ohne zusätzliche Beschichtung zu Beginn der Hochtemperaturexposition immer einen Masseverlust, bevor oberhalb von $1000^{\circ} \mathrm{C}$ Schutz durch die gut fließfähige und schnell wachsende Borosilikat-Glasschicht einsetzt und der Masseverlust gestoppt wird. Die Mechanismen der Oxidation sind in [24] umfänglich dargestellt.

Die $\mathrm{SiO}_{2}$-Schichtbildung gelingt allerdings im Falle der Nb-haltigen Legierungen nicht, da das voluminöse, schnell wachsende und dabei am Substrat haftende $\mathrm{Nb}_{2} \mathrm{O}_{5}$ die Bildung einer dichten $\mathrm{SiO}_{2}$-Schicht unterdrückt, was auch durch die Zugabe von B nicht verhindert werden kann. Deshalb müssen $\mathrm{Nb}$-Silizidlegierungen in der Regel weitere Legierungselemente, wie $\mathrm{Ti}$ (bis über 20 at\%, auch zur weiteren Dichtereduktion), $\mathrm{Cr}$ (bis 15 at.\%) und in geringeren Mengen sogar $\mathrm{Ge}, \mathrm{Sn}$ und $\mathrm{V}$, zugegeben werden, um $\mathrm{Nb}-$ $\mathrm{X}$-O-Mischoxide mit geringeren Wachstumsraten zu erzeugen [25]. Diese weisen jedoch meist lineares Wachstum auf, was auf eine immer noch permeable Schicht hindeutet.

Zusammenfassende Darstellungen über Herstellungsverfahren und den daraus resultierenden Eigenschaften der RM-Silizidlegierungen finden sich in [26-28].

\subsection{Herstellung von Pulver aus Refraktärmetall- siliziden}

Die Herstellung geeigneter Pulver für Halbzeuge aus RMSilizidlegierungen folgt einerseits zunächst der aus der Refraktärmetallherstellung hinlänglich bekannten klassischen Pulvermetallurgie, bestehend aus dem Mischen von Elementpulvern, kalt isostatischem Pressen (engl. Cold Isostatic Pressing, CIP) zum besseren Handling der Grünkörper, und Sintern unter trockenem Wasserstoff (Mo-Basis) bzw. Hochvakuum (Nb-Basis), um hinreichend dichtes Ausgangsmaterial für die Gasverdüsung, wie sie in Abb. 2 bereits beschrieben wurde, zu bekommen. Die Gasverdüsung erfolgt mittels EIGA durch Ar, wie bereits in Abschn. 2.2 erläutert. Zwar sind die wegen der höheren Wärmeleitung erreichbaren Abkühlraten im Vergleich zu TiAl-Legierungen mit bis zu $10^{6} \mathrm{~K} / \mathrm{s}$ höher, allerdings sind die erreichbaren D50-Werte der Pulver wegen der höheren Schmelzpunkte der RM-Silizide mit $70 \mu \mathrm{m}$ (Nb-Basis) bis $100 \mu \mathrm{m}$ (Mo-Basis) 


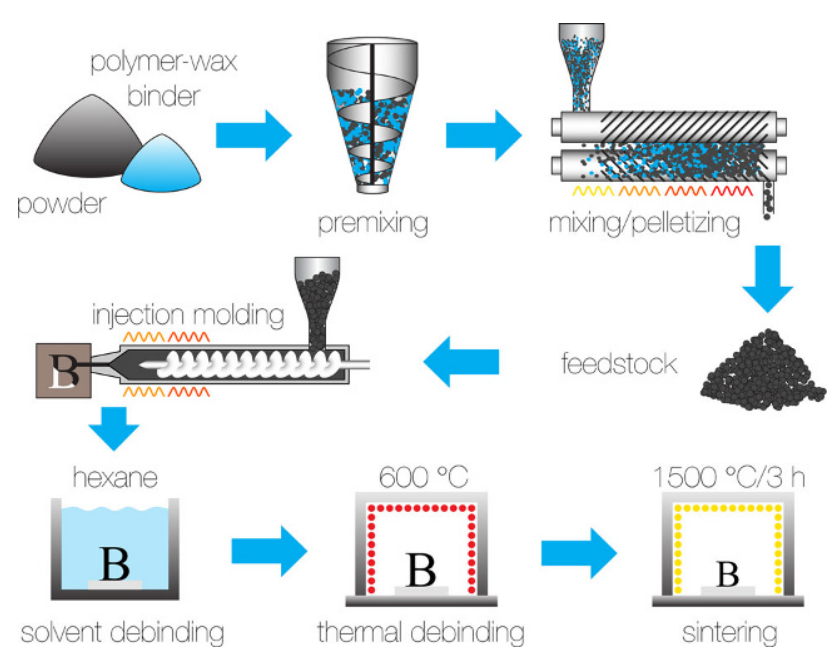

Abb. 4: MIM-Verarbeitungsroute am Beispiel der RM-Silizid-Legierungspulver, siehe auch [33] für weitere Details

auch höher. Weitere Details können in Referenz [29] nachgelesen werden.

Andererseits wurde das Prinzip des mechanischen Legierens (Mechanical Alloying, MA) unter Schutzgasatmosphäre angewendet und auch patentiert [21], hauptsächlich mit dem Ziel, extrem feine und chemisch homogene Pulver herzustellen. Dabei konnte gezeigt werden, dass sich Zustände weitab vom thermodynamischen Gleichgewicht einstellen lassen, die sich insbesondere durch einen stark übersättigten Mischkristall auszeichnen. So wurde im Falle einer Mo-9Si-8B-Legierung gefunden, dass sich nach dem MA im Pulver ein vollständig übersättigter Mischkristall einstellen lässt [30]. Die (metastabilen) einphasigen Pulverpartikel mit einer durchschnittlichen Größe von $50 \mu \mathrm{m}$ sind damit dann gut zu kompakten Probekörpern mit einer Dichte $>99,5 \%$ der theoretischen Dichte durch CIP, Sintern in $\mathrm{H}_{2}$ bei $1600^{\circ} \mathrm{C}$ zur Verringerung der Gasgehalte (hauptsächlich $\mathrm{O}_{2}$ ) und abschließendem HIP bei $1500^{\circ} \mathrm{C}$ und $200 \mathrm{MPa}$ zu verarbeiten [30]. Prinzipiell vergleichbare Befunde wurden auch für die pulvermetallurgische Herstellung von Nb-Silizidlegierungen gefunden [31].

\subsection{Herstellung von Halbzeugen und Bauteilen}

Wie bereits eingangs erwähnt, stellen die hohen Schmelzpunkte der RM-Silizide bis ca. $2000^{\circ} \mathrm{C}$ die Anwendung industriell üblicher Gießrouten vor große Herausforderungen. Für die Herstellung von Halbzeugen und Bauteilen wurden daher bis dato überwiegend Verfahren, die auf der Verarbeitung von Pulvern beruhen, untersucht. Da Umformverfahren wie Walzen oder Extrusion zwar prinzipiell erfolgreich durchgeführt werden konnten, wegen der hierfür erforderlichen hohen Temperaturen bis zu $1600^{\circ} \mathrm{C}$ industriell jedoch schwierig und nur mit speziellen Werkzeugen applizierbar sind [29], konzentrierten sich aktuelle Untersuchungen in dem kürzlich erfolgreich beendeten EU-Projekt HYSOP auf endkonturnahe Fertigungsverfahren [32]. So konnten beispielswiese mittels Net Shape HIP RM-Silizidpulver (sogar) in Stahlkannen bei etwa $1250^{\circ} \mathrm{C}$
(150 MPa, 4h) erfolgreich zu dichten (Restporosität <0,5\%) Leit- und Laufschaufeln bzw. Dichtungselementen unterschiedlicher Geometrien konsolidiert werden, wobei die gasförmigen Verunreinigungen $\mathrm{O}_{2}(2100 \mathrm{ppm})$ bzw. $\mathrm{N}_{2}$ $(<100 \mathrm{ppm})$ auf akzeptabel niedrigem Niveau lagen [32].

Neben "net shape HIPing" wurde im Rahmen des europäischen HYSOP-Projektes [32] auch das bereits in Abschn. 2.2 angesprochene MIM-Verfahren, mit dem sich endkonturnahe Bauteile und Probekörper herstellen lassen, evaluiert, siehe Abb. 4 [33]. Hierzu wird zunächst ein so genannter "Feedstock" aus vorher gasverdüstem oder mechanisch legiertem RM-Silizidpulver mit einem Polymer-basiertem Binder durch Mischen und Pelletieren hergestellt. Pulveranteile bis zu 70\% konnten dabei im Schneckenextrudierer erfolgreich zu einem Grünkörper verarbeitet werden, der zunächst in einem zweistufigen Prozess in flüssigem Hexan und anschließend thermisch bei etwa $600^{\circ} \mathrm{C}$ zu einem handhabbaren Braunkörper entbindert wurde. Für die Nb-20Si-23Ti-3Cr-6Al-4Hf-Legierung führte der finale Sinterprozess unter Vakuum (besser als $10^{-4} \mathrm{mbar}$ ) bei $1500^{\circ} \mathrm{C}$ für $3 \mathrm{~h}$ zu Dichten von $95-97 \%$ der theoretischen Dichte. Die Konzentration der gasförmigen Verunreinigungen lag typisch bei $2700 \mathrm{ppm}\left(\mathrm{O}_{2}\right)$ bzw. $400 \mathrm{ppm}\left(\mathrm{N}_{2}\right)$. Damit sind sowohl die einstellbaren Porositäten als auch die Verunreinigungen mit Gasen im MIM-Prozess vergleichsweise höher als beim Net Shape HIP. Anschließende mechanische Untersuchungen bei Raumtemperatur und Kriechtemperaturen bis $1200^{\circ} \mathrm{C}$ ergaben jedoch keine nennenswerten Unterschiede in den Eigenschaften zwischen MIM- und HIP-Proben, was mit der Vergleichbarkeit der durch die abschließende Wärmebehandlung eingestellten Mikrostrukturen, bestehend aus $\mathrm{Nb}$-Mischkristall und $\mathrm{Nb}_{5} \mathrm{Si}_{3}$-Phase, erklärt werden kann. Zukünftig könnten auch hier, wie bereits im Fall des TiAl erfolgreich praktiziert und in Abschn. 2.3 diskutiert, Verfahren der Generativen Fertigung Erfolg versprechen, da die Frage der Pulverherstellung geklärt scheint.

\section{Zusammenfassung}

Die klassischen Werkstoffe für Triebwerkbauteile, wie Titanlegierungen und Nickellegierungen, haben im Laufe der letzten Jahrzehnte ein hohes Maß an technischer Perfektion erreicht. Obwohl das Verbesserungspotential dieser traditionell verwendeten Werkstoffe begrenzt ist, werden diese Werkstoffe nach wie vor eine wichtige Rolle spielen. Neue Werkstoffe wie intermetallische Titanaluminide auf Basis der geordneten $\gamma$-TiAl Phase und hochschmelzende Refraktärmetallsilizide sind in der Entwicklung bzw. bereits in der Anwendung. Die pulvermetallurgische Herstellung von Bauteilen aus innovativen Werkstoffen bietet eine Vielfalt von Anwendungsmöglichkeiten in modernen Flugzeugtriebwerken und Verbrennungsmotoren. Für die Produktion von hochwertigen Legierungspulvern stehen mehrere industriell relevante Techniken bereit. Auch für die Weiterverarbeitung der Pulver zu Bauteilen stehen mehrere Verfahren zu Verfügung, wobei für die Zukunft speziell dem so genannten Additive Manufacturing eine Schlüsselrolle zukommen wird. 
Danksagung. Die Autoren bedanken sich bei Dr. Bernard Bewlay, Dr. Alain Couret, Dr. Silvia Sabbadini und Dr. Wilfried Smarsly für viele anregende Diskussionen.

Funding. Open access funding provided by Montanuniversity Leoben.

Open Access Dieser Artikel wird unter der Creative Commons Namensnennung 4.0 International Lizenz (http://creativecommons.org/licenses/ by/4.0/deed.de) veröffentlicht, welche die Nutzung, Vervielfältigung, Bearbeitung, Verbreitung und Wiedergabe in jeglichem Medium und Format erlaubt, sofern Sie den/die ursprünglichen Autor(en) und die Quelle ordnungsgemäß nennen, einen Link zur Creative Commons Lizenz beifügen und angeben, ob Änderungen vorgenommen wurden.

\section{Literatur}

1. Triebwerke der Zukunft, www.mtu.de/de/technologie/ zukunftstechnologien/triebwerke-der-zukunft. Zugegriffen: 11.12.2017

2. Appel, F; Paul, J.D.H.; Oehring, M.: Gamma titanium aluminide alloys, Weinheim: Wiley-VCH, 2011

3. Lemberg, J. A., Ritchie, R. O.: Mo-Si-B alloys for ultrahigh-temperature structural applications, Advanced Materials, 24 (2012), S. $3445-3480$

4. Clemens, H.; Mayer, S.: Design, processing, microstructure, properties, and applications of advanced intermetallic TiAl alloys, Advanced Engineering Materials, 15 (2013), S. 191-215

5. Clemens, H.; Smarsly, W.: Light-weight intermetallic titanium aluminides - status of research and development, Advanced Materials Research, 278 (2011), S. 551-556

6. Bewlay, B. P.; Nag, S.; Suzuki, A.; Weimer, M. J.: TiAl alloys in commercial aircraft engines, Mater. High Temp., 33 (2016), S. 549

7. Clemens, H.; Lorich, A.; Eberhardt, N.; Glatz, W.; Knabl, W.; Kestler, $\mathrm{H}$.: Technology, properties and applications of intermetallic $\gamma$-TiAl based alloys, Zeitschrift für Metallkunde,90 (1999), S. 569-580

8. Gerling, R.; Clemens, H.; Schimansky, F. P.: Powder metallurgical processing of intermetallic gamma titanium aluminides, Advanced Engineering Materials, 6 (2004), S. 23-38

9. ALD Vacuum Technologies $\mathrm{GmbH}$ : Powder metallurgy, http://web. ald-vt.de/cms/vakuum-technologie/anlagen/powder-metallurgy/. Zugegriffen: 14.11.2016

10. Schloffer, M.; Iqbal, F.; Gabrisch, H.; Schwaighofer, E.; Schimansky, F.P.; Mayer, S.; Stark, A.; Lippmann, T.; Göken, M.; Pyczak, F.; Clemens, $\mathrm{H}$.: Microstructure development and hardness of a powder metallurgical multi phase $\gamma$-TiAl based alloy, Intermetallics, 22 (2012), S. 231-240

11. Clemens, H., Mayer, S.: Pulvermetallurgie von intermetallischen Titanaluminiden, Berg- und Hüttenmännische Monatshefte (BHM), 160 (2015), S. 513-516

12. Das, G.; Kestler, H.; Clemens, H.; Bartolotta. P. A.: Sheet gamma TiAl: present status and future opportunities, Journal of Metals, 56 (2004), S. 42-45

13. Xu, L.; Wu, J.; Cui, Y.; Yang, R.: Effect of powder pre-treatment on the mechanical properties of powder metallurgy Ti-47Al-2Cr-2Nb0.15B, in: Kim, Y.-W., Smarsly, W., Lin, J., Dimiduk, D., Appel, F (eds.): Gamma Titanium Aluminide Alloys 2014, The Minerals, Metals \& Materials Society, Warrendale, PA, USA, 2014, pp. 195-202

14. Monchoux, J. P.; Voisin, T.; Trzaska, Z.; Durand, L.; Couret, A.; Thomas, M.: One-step near-net shaping of TiAl turbine blades by SPS, Presentation, Gamma Alloy Technology 2013, Toulouse, France, 2013

15. Biamino, S.; Penna, A.; Ackelid, U.; Sabbadini, S.; Tassa, O.; Fino P.; Pavese, M.; Gennaro, P.; Badini, C.: Electron beam melting of Ti48Al-2Cr-2Nb alloy: microstructure and mechanical properties investigation, Intermetallics, 19 (2011), S. 776-781
16. Arcam $A B: E B M \circledast$ in aerospace - additive manufacturing taken to unseen heights, http://www.arcam.com/solutions/aerospace-ebm/. Zugegriffen: 14.11.2016

17. Klöden, B.; Biamino, S.; Ackelid, U.; Weißgärber, T.; Kieback, B.: Titanium aluminides for automotive applications processed by electron beam melting; World PM 2014, CD-Proceedings, Orlando, Florida, USA, 2014

18. Weimer, M.: It took the world (to raise a TiAl LPT blade), presentation at IWTA2016, hosted by Tokyo Institute of Technology, Tokyo, Japan, August 28 - September 2, 2016

19. Berczik, D. M.: Method for Enhancing the Oxidation Resistance of a Molybdenum Alloy and a Method of Making a Molybdenum Alloy, U.S. Patent No. 5,595,616 (January 21, 1997)

20. Jackson, M. R.; Bewlay, B. P.; Zhao, J.-C.: Niobium-silicide based composites resistant to low temperature pesting, U.S. Patent No. 6,419,765 (July 16, 2002)

21. Jehanno, P.; Heilmaier, M.; Kestler, H.: Method for the production of a molybdenum alloy, Europäische Patentschrift, EP 1718777 B1, 2005

22. Schliephake, D.; Azim, M.A; v. Klinski-Wetzel, K.; Gorr, B.; Christ, H.-J.; Bei, H.; George, E.P.; Heilmaier, M.: High-Temperature Creep and Oxidation Behavior of Mo-Si-B Alloys with High Ti Contents, Metall. Mater. Trans., 45A (2014), S. 1102-1111

23. Gang, F; Heilmaier, M.: Influence of Directional Solidification on the Creep Properties of a Binary NbSi Eutectic Alloy, Journal of Metals, 66 (2014) 1908-1913

24. Rioult, F. H.; Imhoff, S. D.; Sakidja, R.; Perepezko, J. H.: Transient oxidation of Mo-Si-B alloys: Effect of the microstructure size scale, Acta Materialia, 57 (2009), S. 4600-4613

25. Chan, K. S.: Cyclic-Oxidation Resistance of Niobium-Base in situ Composites: Modeling and Experimentation, Oxid. Metals, 61 (2004), S. 165-194

26. Dimiduk, D. M.; Perepezko, J. H.: Mo-Si-B Alloys: Developing a Revolutionary Turbine-Engine Material, MRS Bulletin, 28 (2003), S. 639-645

27. Bewlay, B. P.; Jackson; M. R., Zhao, J.-C.; Subramanian, P. R.; Mendiratta, M. G.; Lewandowski, J. J.: Ultrahigh-Temperature Nb-SilicideBased Composites, MRS Bulletin, 28 (2003), S. 639-645

28. Heilmaier, M.; Krüger, M.; Saage, H.; Rösler, J.; Mukherji, D.; Glatzel U.; Völkl, R.; Hüttner, R.; Eggeler, G.; Somsen, G.; Depka, T.; Christ, H.-J.; Gorr, B.; Burk, S.: Metallic Materials for Structural Applications Beyond Nickel-based Superalloys, Journal of Metals, 61 (2009), S. $65-71$

29. Jehanno, P.; Heilmaier, M.; Kestler, H.; Böning, M.; Venskutonis, A.; Bewlay, B. P.; Jackson, M. R.: Assessment of a Powder Metallurgical Processing Route for Refractory Metal Silicide Alloys, Metall. Mater. Trans., 36 A (2005), S. 515-523

30. Krüger, M.; Franz, S.; Saage, H.; Heilmaier, M.; Schneibel, J. H.; Jéhanno, P.; Böning, M.; Kestler, H.: Mechanically alloyed Mo-Si$B$ alloys with a continuous $\alpha-M o$ matrix and improved mechanical properties, Intermetallics, 16 (2008), S. 933-941

31. Seemüller, C.; Hartwig, T.; Mulser, M.; Adkins, N.; Wiggins, M.; Heilmaier, M.: Influence of Powder Metallurgical Processing Routes on Phase Formations in a Multicomponent NbSi-Alloy, Journal of Metals, 66 (2014), S. 1900-1907

32. Hybrid Silicide-Based Lightweight Components for Turbine and Energy (HYSOP), EU 7th Framework Programme, Grant Agreement No. 266214, 2011-2015, http://hysop.onera.fr. Zugegriffen: 05.12.2017

33. Mulser, M.; Hartwig, T.; Seemüller, C.; Heilmaier, M.; Adkins, N. Wiggins, M.: Nb-Si intermetallic composites for high-temperature applications produced by MIM, Proc. Inter. Powder Metallurgy Congress \& Exhibition (Euro PM 2013), EPMA 2013, ISBN 978189907240-8 